\section{INCREDIBLY DURABLE TOOTH-COLOURED WIRES}

Ortho-Care Ltd, one of the UK's largest independent distributors of orthodontic products, has introduced Woowa - an aesthetically pleasing, high-performance archwire, that is incredibly durable.

Ceramic fixed braces may have revolutionised the world of orthodontics but the challenge has always been how to deal with unsightly wires that ruin the 'invisible' look. In the past, the options have been limited. People with cosmetic braces either had to put up with silvercoloured stainless steel wires or resort to poor quality tooth-coloured wires that were prone to peeling.

Woowa archwires are toothcoloured and, when combined with aesthetic brackets, create a truly brace-free look. Developed in Korea, this new technology allows orthodontists to give patients the very best aesthetic orthodontic treatments.
The product does not compromise the clinical performance of their treatment as Woowa wires perform like a noncoated archwire, with outstanding low levels of friction, due to the negligible 0.00005 " coating that does not change the dimensions of the wire. This unique coating can be applied to stainless steel or nickel titanium wire. The outer polymer coating is resistant to corrosion and staining and won't discolour, crack or peel.

Woowa archwires are now available in all popular sizes in nickel titanium and stainless steel.

Reader response number 58

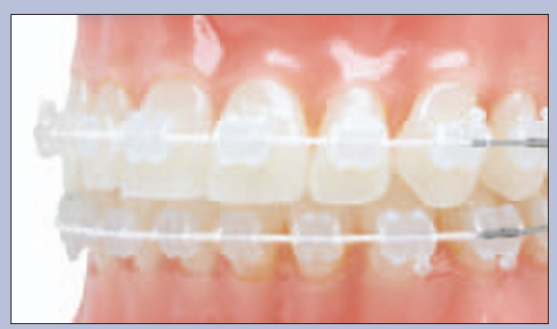

\section{SEE THE COMPLETE PRODUCT RANGE IN ACTION}

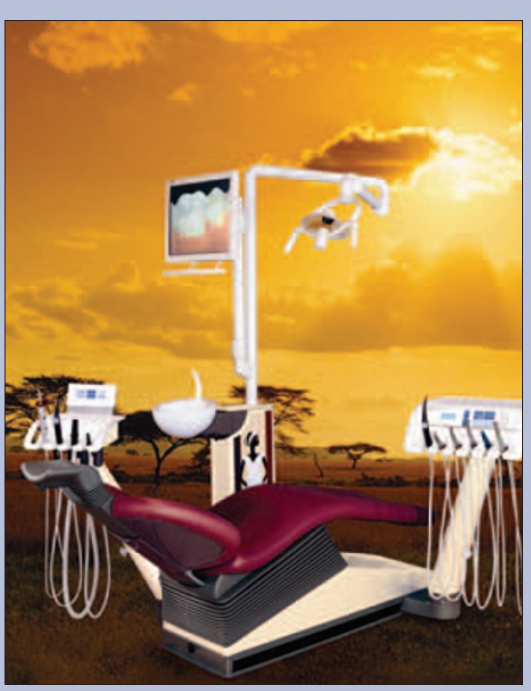

When you buy Sirona Equipment from Sident Dental Systems you get the best price, exclusive special offers and access to the best sales support in the UK. Sident Dental Systems, which has been established 28 years, can offer you: comprehensive bespoke project management services for surgery refurbishments; factory trained engineers; product specialists to advise on design, installation and on-site training; and low cost finance packages for all products.

Sirona Specialists, Sident Dental Systems offer the choice from the complete range of Sirona Treatment Centres, 2D and 3D digital and film based X-ray apparatus - including Galileos 3D digital cone-beam equipment, their extensive range of Sirona handpieces, and auxiliary items including SiroLaser, SIROEndo and DAC sterilisation units. To support these items they offer DURR Suction and Dental Art Cabinetry, as well as other essential equipment from similarly tried and trusted manufacturers.

Wherever possible potential clients are invited to visit The Courtyard, Sident's state-of-the-art training and showroom facility, where they will be able see the complete product range in action.

Reader response number 61 